\title{
Transmural Ventricular Heterogeneities Play a Major Role in Determining T-Wave Morphology at Different Extracellular Potassium Levels
}

\author{
Hassaan A Bukhari ${ }^{1,2,4,5}$, Flavio Palmieri ${ }^{2,3}$, Dina Ferreira ${ }^{8}$, Mark Potse ${ }^{4,5}$, Julia Ramírez ${ }^{6}$, \\ Pablo Laguna ${ }^{1,2}$, Carlos Sánchez ${ }^{1,2,7}$, Esther Pueyo ${ }^{1,2}$ \\ ${ }^{1}$ BSICoS group, I3A Institute, University of Zaragoza, IIS Aragón, Zaragoza, Spain \\ ${ }^{2}$ CIBER en Bioingeniería, Biomateriales y Nanomedicina (CIBER-BBN), Zaragoza, Spain \\ ${ }^{3}$ Centre de Recerca en Enginyeria Biomèdica, Universitat Politècnica de Catalunya, Barcelona, Spain \\ ${ }^{4}$ Carmen team, Inria Bordeaux Sud-Ouest, Talence, France \\ ${ }^{5}$ University of Bordeaux, IMB, UMR 5251, Talence, France \\ ${ }^{6}$ William Harvey Research Institute, Queen Mary University of London, London, United Kingdom \\ ${ }^{7}$ Defence University Centre (CUD), General Military Academy of Zaragoza (AGM), Zaragoza, Spain \\ ${ }^{8}$ Laboratorios Rubio, Castellbisbal, Barcelona, Spain
}

\begin{abstract}
End-stage renal disease (ESRD) affects more than $10 \%$ of the world population. ESRD patients present impaired potassium homeostasis, which increases the risk for ventricular arrhythmias and sudden cardiac death. Noninvasive estimation of serum potassium, $\left[\mathrm{K}^{+}\right]$, before the patient experiences serious consequences is of major importance. In this study, we investigated the relationship of $\left[\mathrm{K}^{+}\right]$with three $T$-wave morphological descriptors: the T-wave width $\left(T_{\mathrm{w}}\right)$, slope-to-amplitude ratio $\left(T_{\mathrm{SA}}\right)$ and temporal morphological variability $\left(d_{\mathrm{w}}\right)$ from ECGs of 12 ESRD patients undergoing hemodialysis and from simulated ECGs. Spearman's correlation coefficients between the descriptors $T_{\mathrm{w}}, T_{\mathrm{SA}}$ and $d_{\mathrm{w}}$ and $\left[\mathrm{K}^{+}\right]$were -0.5 , 0.8 and 0.65 , respectively. These associations were, however, highly patient-dependent. The high inter-individual variability in T-wave morphology, particularly observed at high $\left[\mathrm{K}^{+}\right]$, was reproduced in the simulations and could be explained by differences in transmural heterogeneities, with $10 \%$ variations in the proportion of midmyocardial cells leading to changes larger than $15 \%$ in T-wave morphology. In conclusion, T-wave morphological descriptors have the potential to be used as predictors of $\left[\mathrm{K}^{+}\right]$in ESRD patients, but their associated inter-individual variability should be taken into account, especially under hyperkalemic conditions.
\end{abstract}

\section{Introduction}

End-stage renal disease (ESRD) represents a global health burden, with an estimated $10 \%$ of the worldwide population being affected. This involves high associated economic cost, increased mortality risk, and decreased quality of life for affected patients [1]. As renal function declines, ESRD patients present an increasingly impaired ability to maintain potassium homeostasis. Serum potassium levels $\left(\left[\mathrm{K}^{+}\right]\right)$outside normal ranges, in the form of hypokalemia or hyperkalemia, increase the risk for lifethreatening arrhythmias and sudden cardiac death [2]. Investigating the effects of $\left[\mathrm{K}^{+}\right]$variations on the electrical functioning of the heart could help improve therapies and risk stratification tools, for example by using the ECG to continuously monitor $\left[\mathrm{K}^{+}\right]$.

Variations of $\left[\mathrm{K}^{+}\right]$levels have been shown to affect depolarization and repolarization features of the ECG in previous studies [3-5], suggesting that indeed it is possible to infer $\left[\mathrm{K}^{+}\right]$from the ECG. However, in patient data we found a wide variety of relations between $\left[\mathrm{K}^{+}\right]$and repolarization characteristics in the ECG. These repolarization features are also influenced by the dispersion of repolarization properties in the heart. Therefore we hypothesized that the inter-individual differences in the relation between $\left[\mathrm{K}^{+}\right]$and ECG features can be caused by interindividual differences in the dispersion of primary repolarization properties. We simulated a set of ventricular fibers covering a wide range of transmural heterogeneities and we calculated pseudo-ECGs at different $\left[\mathrm{K}^{+}\right]$. We characterized the simulated T-wave morphology changes by using three descriptors $\left(T_{\mathrm{w}}, T_{\mathrm{SA}}[6]\right.$ and $\left.d_{\mathrm{w}}[7]\right)$ and we compared them with those from ECGs of patients undergoing hemodialysis (HD), i.e. experiencing large $\left[\mathrm{K}^{+}\right]$ variations. Upon confirmation of the model's ability to reproduce inter-individual variability in the evaluated $\mathrm{T}$ wave descriptors, we performed a sensitivity analysis to assess the dependence of T-wave morphology descriptors 
on transmural heterogeneities and assess whether transmural dispersion indeed contributes to explain inter-individual differences in T-wave morphology, particularly for $\left[\mathrm{K}^{+}\right]$ values out of normal ranges.

\section{Methods}

Clinical Measurements The study population included 12 ESRD patients from Hospital Clínico Universitario de Zaragoza (HCUZ). 48-hour 12-lead ECGs were acquired at a sampling frequency of $1 \mathrm{kHz}$ and amplitude resolution of $3.75 \mu \mathrm{V}(\mathrm{H} 12+$, Mortara Instruments, Milwaukee, WI, USA). The acquisition started 5 minutes before the HD treatment onset and lasted for 48 hours (Figure 1 bottom blue line). Simultaneously, six blood samples were taken and analyzed during the HD session: the first one at the HD onset and the next three samples every hour during the HD session (Figure 1, K1 to K4 in red). The $5 t h$ blood sample was collected at the end of the HD (minute 215 or 245 , depending on the patient) while the 6 th blood sample was taken after 48 hours, immediately before the next HD session. Extracellular potassium concentrations, $\left[\mathrm{K}^{+}\right]$, were measured at those time points, as shown in Figure 1. The ethical committee approved the study protocol, with all patients providing signed informed consent.

\begin{tabular}{|c|c|c|c|c|c|}
\hline K1 & $\mathrm{K} 2$ & K3 & K4 & K5 & K6 \\
\hline 05 & 65 & 125 & 185 & 245 & 2880 \\
\hline & \multicolumn{5}{|c|}{ Time(min) } \\
\hline
\end{tabular}

Figure 1. Diagram of the study protocol. K1 to K6 are the time points (in minutes) for blood sample extraction.

The measured ECG signals were band-pass filtered ( 0.5 to $40 \mathrm{~Hz}$ ) to remove baseline wander, muscular noise, and powerline interference. QRS detection and wave delineation were performed by using a wavelet-based singlelead delineation method [8]. To emphasize T-wave components while allowing more accurate delineation, Principal components (PCs) were obtained by computing the auto-correlation matrix of T-waves in a stable ECG segment at the end of the HD session. The ECG recording was subsequently projected into the direction of the 1st PC. The T-waves in the obtained 1st PC were delineated [8] and used for further analysis. In simulated pECGs, singlelead wavelet-based delineation was directly applied over the signals. The onset, peak, and end of the T-wave were determined.

Computational Modeling Transmural electrical propagation from ventricular endocardium to epicardium was simulated using 1-dimensional fibers $(1.65 \mathrm{~cm})$. A train of 10 stimuli was applied to the first cell of the fiber every $1000 \mathrm{~ms}$ with an amplitude equal to 1.5 times the diastolic threshold. Cellular electrophysiology was represented by a modified version of the O'Hara model [9] for the human ventricular action potential, as described by Dutta et al. [10]. To compute electrical propagation, a finite element method-based software [11] was used with a time step of $0.5 \mathrm{~ms}$ and space discretization of $0.01 \mathrm{~cm}$. Intracellular and extracellular conductivities in the fiber were adjusted to obtain a conduction velocity of $44 \mathrm{~cm} / \mathrm{s}$.

Different proportions of endo-, mid- and epicardial cells were simulated for a total of 19 combinations: endocardial layer ranging from $10 \%$ to $50 \%$, midmyocardial layer from $10 \%$ to $40 \%$ and epicardial layer from $20 \%$ to $80 \%$. We use the notation CXYZ, where $\mathrm{C}$ stands for the word "case" and X, Y and Z denote the first digit of the proportions of endocardial, midmyocardial and epicardial cells, respectively (e.g. C532 represents the case with 50\%, 30\% and $20 \%$ of endocardial, midmyocardial and epicardial cells, respectively).

Pseudo-electrocardiogram signals (pECGs) were computed using equation (1) as described by Gima et al. [12]:

$$
V_{\mathrm{e}}=\epsilon \int \nabla V_{\mathrm{m}} \cdot\left(\nabla \frac{1}{r}\right) d r,
$$

where $\epsilon$ is a constant proportional to the ratio of intracellular and extracellular conductivities, $V_{\mathrm{m}}$ is the transmembrane potential and $r$ is the distance between each cell in the fiber and the virtual electrode located $2 \mathrm{~cm}$ away from the last epicardial cell in the fiber direction.

Simulations for each ventricular fiber and associated pECGs were run for five different values of $\left[\mathrm{K}^{+}\right]$, which included the reference physiological level in the O'Hara model, i.e. $\left[\mathrm{K}^{+}\right]=5.4 \mathrm{mmol} / \mathrm{l}$, as well as two levels below and two above it: $\left[\mathrm{K}^{+}\right]=2,3,5.4,7,8 \mathrm{mmol} / \mathrm{l}$.

T-wave Morphology Characterization The following T-wave morphological descriptors were studied:

- $T_{\mathrm{w}}$, representing the width of the T-wave from T-wave onset to T-wave end;

- $T_{\mathrm{SA}}$, representing the ratio between the T-wave downward slope and amplitude, calculated as in [6]; and

- $d_{\mathrm{w}}$, representing temporal variations in T-wave morphology [7] (Figure 2).

$d_{\mathrm{w}}$ was computed considering as a reference T-wave the T-wave measured at physiological $\left[K^{+}\right]=5.4 \mathrm{mmol} / \mathrm{L}$ in the simulations and the T-wave measured at the end of the HD session in ESRD patients. Let's now consider the T-wave for a given $\left[\mathrm{K}^{+}\right](\mathrm{mmol} / \mathrm{L}), \boldsymbol{f}^{s}\left(\boldsymbol{t}^{s}\right)=$ $\left[f^{s}\left(t^{s}(1)\right), \ldots, f^{s}\left(t^{s}\left(N_{s}\right)\right)\right]^{\top}$, and the reference $\mathrm{T}$ wave, $\boldsymbol{f}^{r}\left(\boldsymbol{t}^{r}\right)=\left[f^{r}\left(t^{r}(1)\right), \ldots, f^{r}\left(t^{r}\left(N_{r}\right)\right)\right]^{\top}$, where $\boldsymbol{t}^{r}=$ $\left[t^{r}(1), \ldots, t^{r}\left(N_{r}\right)\right]^{\top}$ and $\boldsymbol{t}^{s}=\left[t^{s}(1), \ldots, t^{s}\left(N_{s}\right)\right]^{\top}$ and $N_{r}$ and $N_{s}$ are the total durations of $\boldsymbol{t}^{r}$ and $\boldsymbol{t}^{s}$. Figure 2 (top left panel) shows $\boldsymbol{f}^{r}$ and $\boldsymbol{f}^{s}$, with their respective time domains, $\boldsymbol{t}^{r}$ and $\boldsymbol{t}^{s}$. Let $\gamma\left(\boldsymbol{t}^{r}\right)$ be the warping function that 

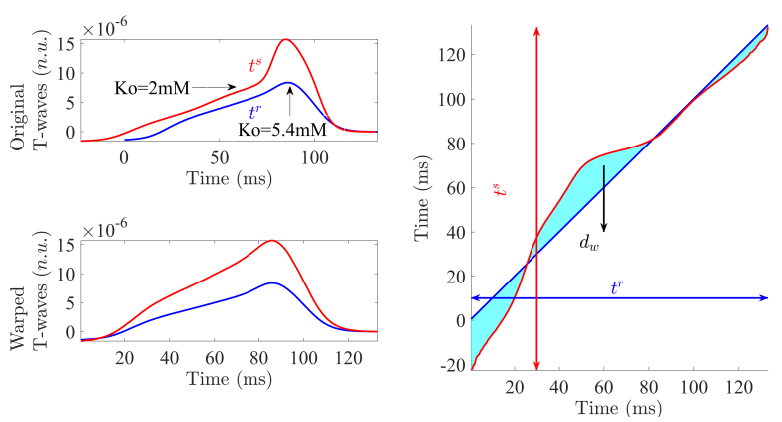

Figure 2. Time warping analysis for simulated fiber $\mathrm{C} 118$. Top left panel shows reference (blue) and studied (red) Twaves aligned with respect to their gravity centers. The blue area in the right panel represents $d_{\mathrm{w}}$. Bottom left panel depicts the final warped T-waves.

relates $\boldsymbol{t}^{r}$ and $\boldsymbol{t}^{s}$, such that $\boldsymbol{f}^{s}\left(\gamma\left(\boldsymbol{t}^{r}\right)\right)$ denotes the timedomain warping of $\boldsymbol{f}^{s}\left(\boldsymbol{t}^{s}\right)$ using $\gamma\left(\boldsymbol{t}^{r}\right)$. The square-root slope function (SRSF) transformation is defined as:

$$
\boldsymbol{q}_{f}(\boldsymbol{t})=\operatorname{sign}(\dot{\boldsymbol{f}}(\boldsymbol{t}))|\dot{\boldsymbol{f}}(\boldsymbol{t})|^{\frac{1}{2}} .
$$

The optimal warping function is the one that minimizes the SRSF amplitude difference:

$$
\gamma^{*}\left(\boldsymbol{t}^{r}\right)=\underset{\gamma\left(\boldsymbol{t}^{r}\right)}{\arg \min }\left(\left\|\boldsymbol{q}_{f^{r}}\left(\boldsymbol{t}^{r}\right)-\boldsymbol{q}_{f^{s}}\left(\gamma\left(\boldsymbol{t}^{r}\right)\right) \sqrt{\dot{\gamma}\left(\boldsymbol{t}^{r}\right)}\right\|\right) .
$$

A dynamic programming algorithm was used to obtain the optimal warping function, $\gamma^{*}\left(\boldsymbol{t}^{r}\right)$, that optimally warps $\boldsymbol{f}^{r}\left(\boldsymbol{t}^{r}\right)$ and $\boldsymbol{f}^{s}\left(\boldsymbol{t}^{s}\right)$, which is shown in Figure 2 (right panel). The warped T-wave, $\boldsymbol{f}^{s}\left(\gamma^{*}\left(\boldsymbol{t}^{r}\right)\right)$, is shown in Figure 2 (bottom left panel), together with the reference Twave, $\boldsymbol{f}^{r}\left(\boldsymbol{t}^{r}\right)$.

The descriptor $d_{w}$ (Figure 2, right panel) quantifies the level of warping required to optimally align two T-waves:

$$
d_{w}=\left(\frac{s_{d}}{\left|s_{d}\right|}\right) \frac{1}{N_{r}} \sum_{n=1}^{N_{r}}\left|\gamma^{*}\left(t^{r}(n)\right)-t^{r}(n)\right|,
$$

where $s_{d}=\sum_{n=1}^{N_{r}^{u}}\left(\gamma^{*}\left(t^{r}(n)\right)-t^{r}(n)\right)$ is used to account for the sign estimated at the $N_{r}^{u}$ T-wave upslope samples.

Sensitivity Analysis To assess how the proportion of endo-, mid- and epicardial cells modulated T-wave morphology descriptors at different $\left[\mathrm{K}^{+}\right]$, a sensitivity analysis was performed. For each T-wave descriptor, the percentage of change and its sensitivity to changes in the proportion of cells of each ventricular layer were computed as follows [13]:

$$
D_{m ; p ; a_{\mathrm{i}}}=\left(\frac{M_{p ; a_{\mathrm{i}}}-M_{\text {control }}}{M_{\text {control }}}\right) \times 100, \quad i=1,2
$$

$$
S_{m ; p ; a 1, a 2}=\left(\frac{D_{m ; p ; a_{2}}-D_{m ; p ; a_{1}}}{a_{2}-a_{1}}\right) \times 100
$$

where $M_{\mathrm{p} ; \mathrm{a}}$ is the value of the T-wave descriptor $(m)$ under a varying proportion $a$ of cells in the analyzed layer $(p)$ calculated at proportions $a_{1}$ and $a_{2} . M_{\text {control }}$ is the value of the T-wave descriptor at the default proportion of cells in the layer, this being 30,30 and 40\% for endocardial, midmyocardial and epicardial cells, respectively.

\section{Results and Discussion}

Figure 3 illustrates the results of the T-wave characterization for one of the ventricular fibers simulated in this study. Panel (a) shows the aligned T-waves corresponding to $\left[\mathrm{K}^{+}\right]$ranging from 2 to $8 \mathrm{mmol} / \mathrm{L}$. Panels (b), (c) and (d) show the relationship between each of $T_{\mathrm{w}}, T_{\mathrm{SA}}$ and $d_{\mathrm{w}}$ and $\left[\mathrm{K}^{+}\right]$, respectively. $\left[\mathrm{K}^{+}\right]$elevation leads to a reduction in the T-wave duration, an increase in the ratio between $\mathrm{T}$-wave slope and amplitude and enhancement of T-wave temporal morphological variability.

Figure 4 illustrates the T-wave descriptors $T_{\mathrm{w}}, T_{\mathrm{SA}}$ and $d_{\mathrm{w}}$ for four simulated fibers (top Panel (a)) and four patients of the study population (bottom panel (b)) as a function of $\left[\mathrm{K}^{+}\right]$, respectively. Although the three T-wave morphology descriptors are correlated with $\left[\mathrm{K}^{+}\right]$(median Spearman's correlation coefficients of $-0.5,0.8$ and 0.65 ), a diversity of patterns in the three relationships is apparent, both in simulated and patients' ECGs.

Figure 5 shows the results from the sensitivity analysis for $T_{\mathrm{SA}}$. Analogous results were obtained for $T_{\mathrm{w}}$ and $d_{\mathrm{w}}$. As can be observed, the largest sensitivity of $T_{\mathrm{SA}}$ was found to variations in the proportion of midmyocardial cells within the ventricular wall. This was particularly more prominent when $\left[\mathrm{K}^{+}\right]$became elevated above physiological levels. For $\left[\mathrm{K}^{+}\right]=8 \mathrm{mmol} / \mathrm{l}$, sensitivity values above $150 \%$ were found, which means that $10 \%$ variations in the midmyocardial proportion led to changes larger than $15 \%$ in $T_{\mathrm{SA}}$ (similarly for the other T-wave descriptors).

Descriptors of T-wave width, amplitude-to-slope ratio, and temporal morphological variability were shown to vary with $\left[\mathrm{K}^{+}\right]$in both simulated and measured ECGs, but a wide range of patterns was observed for such relationships. The proportion of midmyocardial cells within the simulated ventricular fibers has a large impact on T-wave morphology descriptors, particularly under elevated $\left[\mathrm{K}^{+}\right]$. This suggests that transmural heterogeneities can have a relevant role in predicting the patient-specific response of ventricular repolarization to hyperkalemia.

\section{Acknowledgements}

This work was supported by projects ERC-2014-StG 638284 (ERC), DPI2016-75458-R (MINECO), Marie Skłodowska-Curie grant 764738 and Aragón Government 

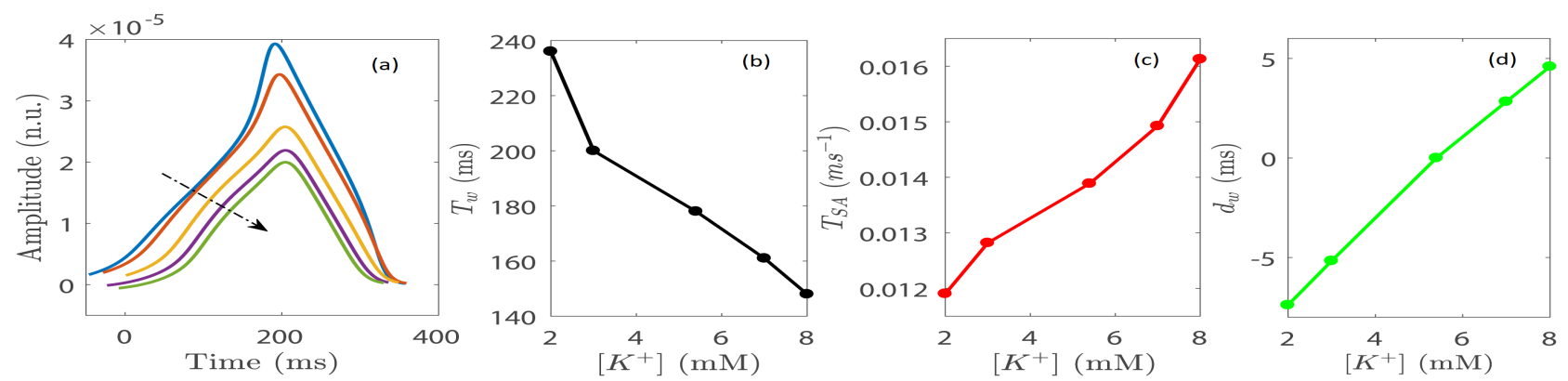

Figure 3. T-waves and $T_{\mathrm{w}}, T_{\mathrm{SA}}$ and $d_{\mathrm{w}}$ evaluated for simulated fiber $\mathrm{C} 145$ under varying $\left[\mathrm{K}^{+}\right]$. The arrow in the left panel indicates the direction of increasing $\left[\mathrm{K}^{+}\right]$from 2 to $8 \mathrm{mmol} / \mathrm{L}$.

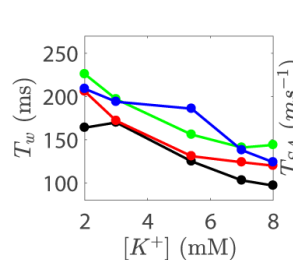

(a) Simulated Results

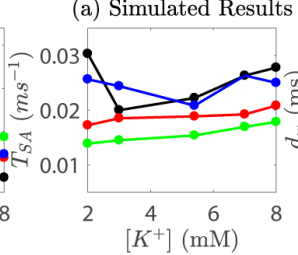

(b) HD ECG Results
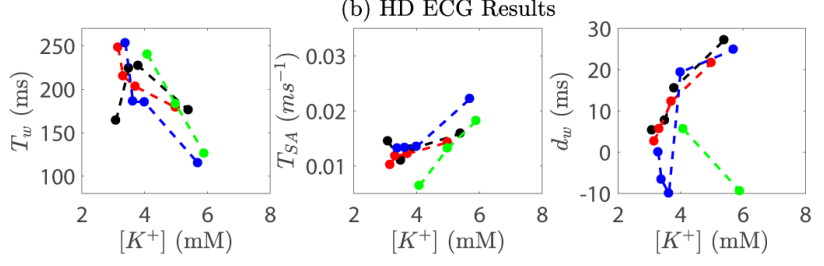

Figure 4. $T_{\mathrm{w}}, T_{\mathrm{SA}}$ and $d_{\mathrm{w}}$ for simulated fibers C118, C127, C136 and C532 and patients HCUZP1, HCUZP4, HCUZP8 and HCUZP15 at varying $\left[\mathrm{K}^{+}\right]$.
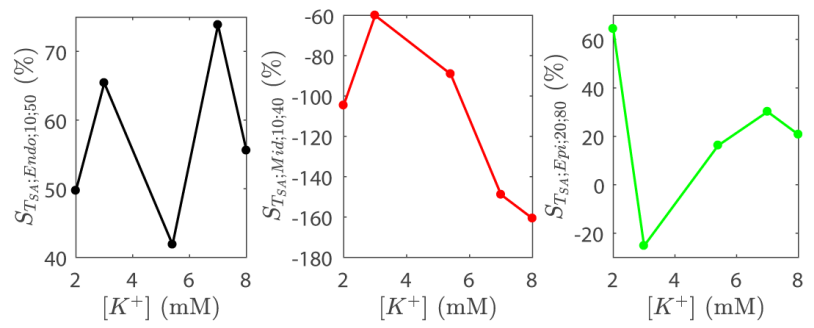

Figure 5. Sensitivity analysis for $T_{\mathrm{SA}}$ to variations in the proportion of endocardial (left), midmyocardial (middle) and epicardial (right) layers at varying $\left[\mathrm{K}^{+}\right]$.

(Reference Group BSICoS T39-17R) cofunded by FEDER 2014-2020. JR acknowledges support from EU H2020 under Marie Sklodowska-Curie grant 786833.

\section{References}

[1] Hill NR, et al. Global prevalence of chronic kidney disease a systematic review and meta-analysis. PLoS ONE July 2016;11(7):e0158765.

[2] Weiss JN, et al. Electrophysiology of hypokalemia and hy- perkalemia. Circ Arrhythm Electrophysiol Mar. 2017;10:110 .

[3] Lanari A, et al. Electrocardiographic effects of potassium. I. Perfusion through the coronary bed. Am Heart J Mar. 1964;67(3):357-363.

[4] El-Sherif N, et al. Electrolyte disorders and arrhythmogenesis. Cardiol J 2011;18(2):233-245.

[5] Mieghem C, et al. The clinical value of the ECG in noncardiac conditions. Chest Apr. 2004;125(4):1561-1576.

[6] Corsi C, et al. Validation of a novel method for non-invasive blood potassium quantification from the ECG. Computers in Cardiology Sep. 2012;39:105-108.

[7] Ramírez J, et al. Variability of ventricular repolarization dispersion quantified by time-warping the morphology of the T-wave. IEEE Trans Biomed Eng Jul 2017;64(7):16191630.

[8] Martínez JP, et al. A wavelet-based ECG delineator: Evaluation on standard databases. IEEE Trans Biomed Eng Apr. 2004;4(51):570-581.

[9] O'Hara T, et al. Simulation of the undiseased human cardiac ventricular action potential: model formulation and experimental validation. PLoS Comput Biol May 2011; 7(5):e1002061.

[10] Dutta S, et al. Electrophysiological properties of computational human ventricular cell action potential models under acute ischemic conditions. Progress in Biophysics and Molecular Biology Oct. 2017;129:40-52.

[11] Heidenreich EA, et al. Adaptive macro finite elements for the numerical solution of monodomain equations in cardiac electrophysiology. Ann Biomed Eng Mar. 2010; 38(7):2331-45.

[12] Gima K, et al. Ionic current basis of electrocardiographic waveforms a model study. Circ Res May 2002;90(8).

[13] Romero L, et al. Impact of ionic current variability on human ventricular cellular electrophysiology. Am J Physiol Heart Circ Physiol Oct. 2009;297(4):1436-1445.

Address for correspondence:

Hassaan A. Bukhari; Institute of Engineering Research of Aragón, Universidad de Zaragoza (Spain).

hassaanahmed01@unizar.es 